\title{
Immunotherapy in lung cancer in times of the COVID-19 pandemic
}

\author{
G V Schiappacasse Cocio* \\ Chief Physician, Oncology Department, Clinical Hospital of Viña del Mar, Chile
}

*Corresponding author: GV Schiappacasse Cocio, Chief Physician, Oncology Department, Clinical Hospital of Viña del Mar, Chile

\begin{abstract}
The current pandemic due to COVID-19 has become a dilemma in decision-making in clinical oncology. On the one hand, the mortality associated with this viral infection is significantly higher in patients with cancer, especially in lung cancer. On the other hand, it is well-known that immunotherapy (PD-1 blockade) improves survival in advanced lung cancer. In the present view point, we aim to clarify the procedure to follow in the context of this pandemic. We conclude that immunotherapy does not increase the risk of serious events produced by COVID-19 in patients with lung cancer. Then, immunotherapy should not be delayed or stopped in patients with lung cancer in times of viral pandemic.
\end{abstract}

Keywords: Immunotherapy; PD-1 Blockade; Pandemic; COVID-19; Lung Cancer

\section{Introduction}

In the context of the current COVID-19 pandemic, a significant part of the medical community has taken the decision to postpone or suspend oncology therapies with clinical benefit. Here, clinical benefit should be understood as therapies which improve the quality of life and survival in cancer patients. For instance, in a prospective study in patients with lung cancer but negative COVID-19 $(n=211)$, the corresponding palliative therapy was delayed (interrupted) in the $39.7 \%$ (3\%) of cases [1]. At the beginning of the pandemic, we may understand this kind of decision due to the insufficient knowledge about the virus behavior in cancer patients. Today, almost one year after the onset of the viral infection, knowledge about this issue has drastically increased.

\section{The Dilemma is the Following}

a) The COVID-19 infection significantly increases mortality in cancer patients. Especially in cases of lung cancer. A systemic review ( $\mathrm{n}=52$ studies with 18,650 cases) reveals that patients with cancer and COVID-19 infection have a higher mortality than the COVID-19 infected population without cancer (mortality of $25.6 \%$ ). Cancer is an independent risk factor for mortality (especially hematologic malignancies and lung cancer). Other risk factors are age, the male gender, the black race, current smoking habit, and comorbidities [2]. b) The immunotherapy (PD-1 blockade) significantly improves survival in advanced lung cancer patients $[3,4]$. Taking into account the clinical benefit of PD-1 blockade and the COVID-19 scenario, the question to be faced is the following: Should immunotherapy be postponed or stopped in patients with advanced lung cancer? The answer to this question is of utmost importance because it allows us to make a decision in routine oncological practice. This decision will have a direct impact on the morbidity and mortality of this type of patients. The article "Impact of PD-1 Blockade on Severity of COVID-19 in Patients with Lung Cancers" concludes that in patients with lung cancer and positive COVID-19 (through RT-PCR in nose or throat smears), the use of PD-1 blockade, before the diagnosis of viral infection, does not increase the risk of severe events produced by COVID-19. There was also no significant difference in serious events produced by COVID-19 in patients with lung cancer in relation to those with recent or past exposure to immunotherapy [5].

\section{The Limitations of the Article are the Following}

a) Retrospective study

b) Single-center study 
c) Small sample size ( $\mathrm{n}=69$ cases $)$

d) The short follow-up from diagnosis of COVID-19 (14 days).

\section{The Strengths of the Article are}

a) The study was approved by an ethics committee

b) The primary question of the study was defined (the impact of PD-1 blockade on severity of COVID-19 in patients with lung cancer)

c) Diagnosis of COVID-19 infection by laboratory test considered the gold standard

d) Exclusion of suspected but unconfirmed cases

e) Suitable definition of what is a serious event by COVID-19

f) Appropriate methodological design

g) Risk factor adjustment for severe event produced by COVID-19 in lung cancer (smoking status and gender)

h) Various data sources were used

i) The data record was exhaustive

j) Expert human resource in data collection was used

k) An independent reviewer was used to give more reliability to the data collection

l) Correct statistical analysis

m) Updated and relevant bibliographic support

n) The primary question of the study was answered

According to our analysis, the strengths of this study are greater than its limitations. Therefore, the conclusion of the article is valid. However, a valid conclusion is not necessarily sufficient for decision making.

We Consider that the Mentioned Conclusion is not Sufficient Mainly Because

a) The analyzed article does not have enough statistical weight (the sample size is limited, $n=69$ )

b) And the study is a retrospective one

\section{As a Result, we Should Complement the Discussion}

The article "COVID-19 mortality in patients with cancer on chemotherapy or other anticancer treatments: a prospective cohort study" is a prospective observational study ( $\mathrm{n}=800$ cases), which considers cancer patients who test positive for COVID-19. After adjusting for other risk factors, there was no significant increase in mortality between the group that received immunotherapy within 4 weeks of the diagnosis of COVID-19 versus the group that did not receive this treatment [6]. Thus, based on the best available evidence, we conclude that the immunotherapy (PD-1 blockade) in lung cancer should not be postponed or suspended because it does not increase critical events due to the COVID-19 viral infection. Postponing or suspending immunotherapy would increase morbidity and mortality from lung cancer. However, it should not be forgotten that we must strictly maintain the safeguard measures to reduce the risk of contagion.

\section{Acknowledgements}

Does not apply.

\section{References}

1. Elkrief A, Kazabdjian S, Bougamin N (2020) Changes in lung cancer treatment as a result of the COVID-19 pandemic: a prospective observational study. JAMA Oncol.

2. Saini KS, Tagliamento M, Lambertini M, McNally R, Romano M, et al. (2020) Mortality in patients with cancer and coronavirus disease 2019. A systematic review and pooled analysis of 52 studies. European Journal of Cancer 139: 43-50.

3. Scott JA, Augusto V, Davey D, David V, Shuji M, et al. (2018) Overall Survival with Durvalumab after Chemoradiotherapy in Stage III NSCLC. N Engl J Med 379(24): 2342-2350.

4. Mok TS, Wu YL, Kudaba I, Kowalski DM, Cho BCh, et al. (2019) Pembrolizumab versus chemotherapy for previously untreated, PD-L1expressing, locally advanced or metastasic non-small-cell lung cancer (KEYNOTE-042): a randomized, open-label, controlled, phase 3 trial. The Lancet 393(10183): 1819-1830.

5. Luo J, Rizvi H, Egger JV, Preeshagul IR, Wolchok JD, et al. (2020) Impact of PD-1 Blockade on Severity of COVID-19 in Patients with Lung Cancers. Cancer Discov. 10(8): 1121-1128.

6. Lee LY, Cazier JB, Angelis V, Arnold R, Bisth V, et al. (2020) COVID-19 mortality in patients with cancer on chemotherapy or other anticancer treatments: a prospective cohort study. Lancet 395(10241): 1919-1926.

\section{OAJOM}

DOI: $10.32474 /$ OAJOM.2020.04.000182

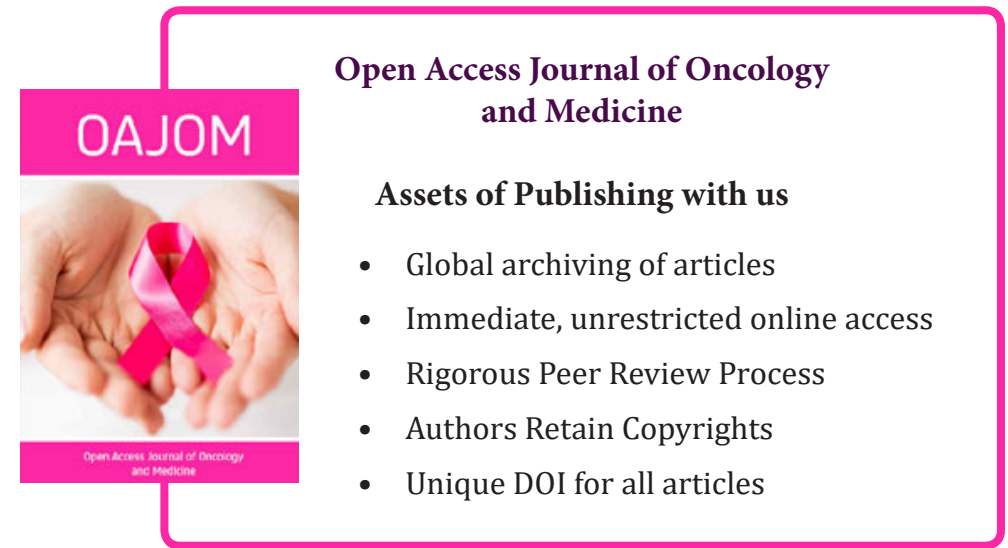

